\title{
Integration of GPS and Cellular Technologies for Development of Smart Public Transport Network
}

\author{
Rajani Mohan Suryavanshi \\ Department of Computer Engineering \\ MIT College of Engineering \\ Pune-411038, India
}

\author{
Rohan Koul \\ Department of Computer Engineering \\ MIT College of Engineering \\ Pune-411038, India
}

\begin{abstract}
The rate of traffic problems and accidents has increased rapidly in the past few years. With the growth in population, the number of vehicles on the road have also increased significantly which in turn contributes to the increasing complexity associated with the traffic management especially in the Public Transport Network. Intelligent Transportation System (ITS) provides solution to most of these problems by integrating existing technologies with the underlying infrastructure. With the advent of GPS and the ubiquitous cellular network like VANET and DSRC, real time vehicle tracking for better public transport management has become possible. Precious time is wasted in long waits at the bus terminus, unaware about when exactly the bus will arrive. This confusion can be obviated by Smart Public Transportation System. Here a methodology based on combination of GPS and GSM/GPRS modem is discussed to help the people who avail the public transportation as their means of travelling. With the details provided by this system, the commuter can plan their journey in advance, hence making public transit a desirable option.
\end{abstract}

\section{General Terms}

Intelligent Transportation System, GPS , DSRC

\section{Keywords}

GSM , GPS , Server , ITS, DSRC

\section{INTRODUCTION}

Public transport is a service that is available on shared basis. This shared service is mostly utilized by the passengers who are the general public, the common people. Mass transit is more efficient, cheaper and occasionally faster than private vehicles. Private vehicles involve a lot of expenditure on gas/oil, a commodity whose prices are soaring day by day, coupled with congestion and limited number of parking spots available. All these problems make Public Transport a very viable and an attractive option. The majority of the Green House Gas emissions from the transportation industry are due to traffic jams and congestion which is a direct consequence of ever increasing number of private vehicles. Congestion is a man-made urban problem that can be rectified using Smart Public Transportation Network.

Turning to the other side of the argument, public transportation has some shortcomings as well. Because of the uncertainty in the bus arrival time, long waits for the arrival of the buses is one of the biggest disadvantages of using public transit. Buses have a fixed scheduled timetable, but to our disappointment the timetable is not followed most of the time. The buses do not arrive on time , leading to delay in the schedules of all the waiting commuters. Urbanization, that is the population shift from rural to urban areas, results in expansion of cities because of which, the number of buses as well as theirs routes are increasing. This expansion adds further to the complexity of the existing transport network, creating confusion amongst the commuters and causing more problems. Referring to all problems mentioned above, the knowledge of bus information like the time of arrival at each bus stop can solve them to a very large extent. In order to provide worthwhile bus data to all passengers, this paper proposes Smart Public Transport System where all required bus information will be gathered, processed, and presented to the users in an efficient and accurate manner. In addition, there will also be a section that will alert the driver when the bus over speeds and record the bus details, location, date and time. These recordings will help authorities to maintain logs and take necessary action against those that are at fault.

Along with the uncertainty regarding the arrival of bus ,there is also apprehension regarding the capacity of bus. Even if the passenger is aware about the arrival time of the bus, if he is not aware about how much more people can be accomodated inside the bus, the information will be half- baked and of no use .Hence the proposed system can eradicate the uncertainity in arrival time commuters face everyday as well prove a great aid in planning their journeys in advance. At the same time the system can allow a better allocation of public funding, maximize use and efficiency of existing infrastructure and improve environmental sustainability. The paper is organized as follows : Section II of the paper discusses about the development of ITS in different parts of the world .Section III describes the underlying technologies that can be integrated with the existing transport network to make it smarter. Section IV talks about the proposed architecture, an innovative approach consisting of three simple modules and also describes how participatory sensing can contribute in increasing the efficiency of the system. Section V and Section VI deals with conclusion and future enhancements to the proposed architecture. 


\section{THE DEVELOPMENT OF ITS IN VARIOUS COUNTRIES}

Intelligent Transportation System (ITS) is based on the increasing demands of the transportation industry and the nuisance caused by traffic jams, congestion and carbon emissions . It integrates information, communications, computers and other technologies and applies them in the field of transportation to build an integrated system of people, roads and vehicles by utilizing advanced data communication technologies. It can establish a large, full-functioning, real- time, accurate and efficient transportation management system. ITS has been implemented in different countries using varied approaches . All these approaches ultimately narrow down to solve the issue of traffic congestion. Each implementation is unique in its own way depending upon the level of prevalent traffic congestion as well as other local conditions. This section presents an analysis of the various implementations of ITS in the developed countries like USA, Japan and Singapore .

\subsection{Japan}

Because of Japanese government's massive investment on ITS, they have got impressive benefits from applying those technologies to the underlying infrastructure of transportation. The maturity of these applications enables Japan to play a pioneering role in Intelligent Transportation Systems across the globe.

To collect and transmit real-time traffic information, Japan began their research in 1996 and created the world's first vehicle information communications system (VICS)[1] which has been available in nationwide since 2003. Japan's VICS takes information collected by Japan's Road Traffic Information Center on roadway conditions, accidents, congestion, and road closures ; processes, edits, and digitizes this information; and then sends it to vehicle navigation systems via different transmission mechanisms. Finally the extracted information is sent onto the driver's car navigation unit in one of three ways: text, simple graphics, or map. Japanese driver's VICSenabled on-board car navigation systems dynamically process the VICS data and suggest to the driver optimal route guidance to avoid accidents, congestion, weather, and/or roadway hazards. VICS thus provides drivers with in-vehicle, real-time traffic information and can assist the driver in selecting as well as plotting an optimal route to get to his or her destination[2]. In the similar manner VICS in-

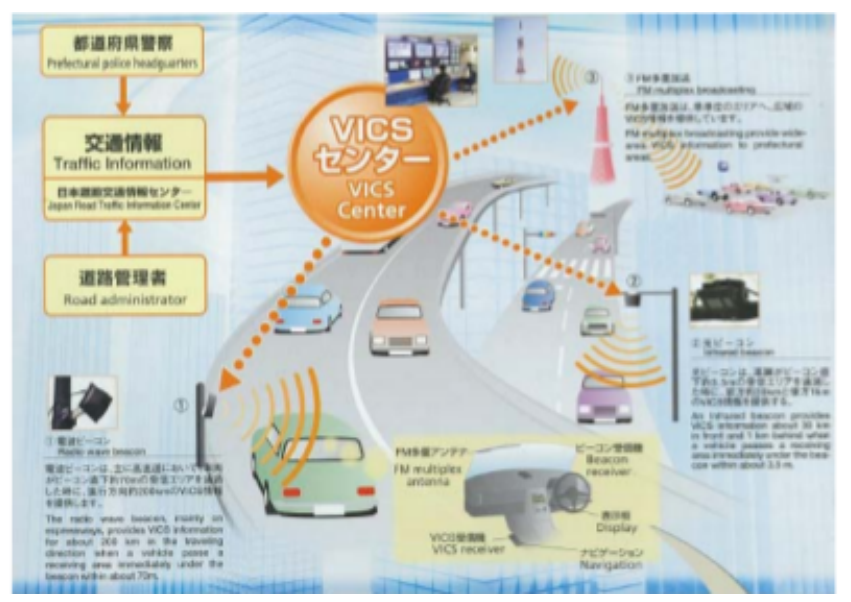

Fig. 1. Japan's Vehicle Information Communications System (VICS) formation is transmitted to motorists in one of three ways:

(1) When a vehicle passes under radio wave beacons, which is installed on Japan's expressways,providing traffic information for about $200 \mathrm{~km}$ in front of the vehicles position.

(2) via infrared beacons which provide VICS information for about $30 \mathrm{~km}$ ahead of the vehicles position and

(3) via FM multiplex broadcasting, which provides wide-area VICS information to prefectural areas.The VICS system uses $5.8 \mathrm{GHz}$ DSRC technology.

The technical architecture of Japan's VICS (Fig. 1) which was designed in the early 1990s was called Version 1.0 of in-car navigation systems in Japan. Japan is developing the Smartway now, which might be called Version 2.0 of the country's state-of-the-art ITS service [2]. Smartway finished its concept development in 2004 and was put into partial deployment in 2007. This process only took 3 years. It is an extremely fast development timeline. Japan began widespread national Smartway deployment in 2010[2].Smartway cooperated with vehicle-highway was developed on the basis of Japan's deployment of ITS experience. As shown in in Fig. 2 , overall design of Smartway, there are a lot of services which are provided by using a platform. Using $5.8 \mathrm{GHz}$ DSRC technology, Smartway can provide visual information of road conditions ahead, traffic information through audio in a visual format and location, and contextually specific information to the driver. Smartway is able to warn drivers when they are nearby across particularly accident prone zones of a roadway, and uses a DSRC-enabled roadside unit to alert drivers plying on the main lanes of the presence of merging vehicles and also sends appropriate warnings regarding the same.

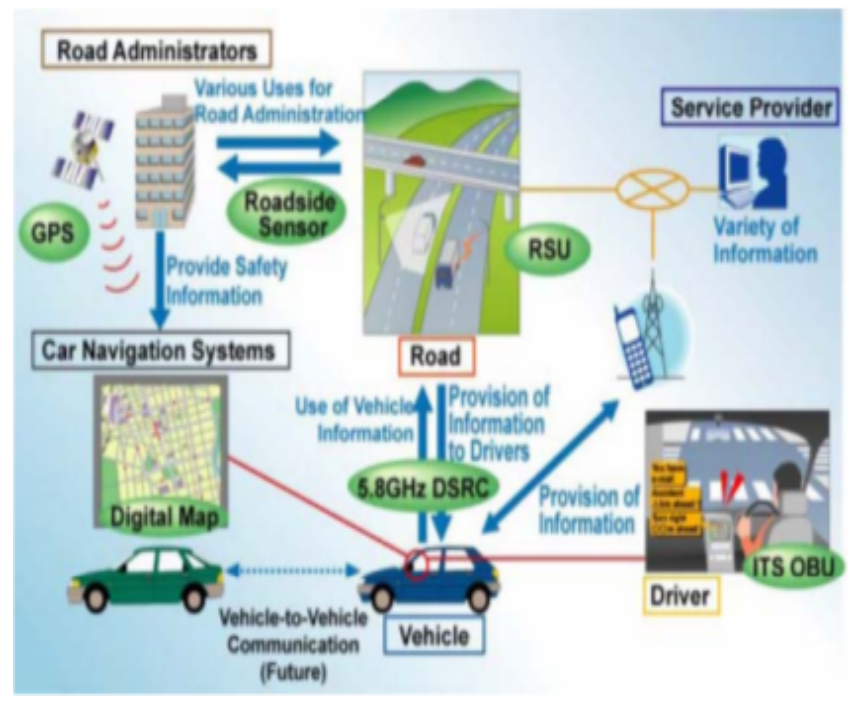

Fig. 2. Overall architecture of Smartway in Japan

\subsection{U.S.A}

In the United States, Electronic Route Guidance System (EGRS) was the initial stage of Intelligent Transportation sys tem (ITS) in 1970s. In 1991 the United States Congress enacted integrated surface transportation efficiency programs (ISTEA). TEA-21 (Transportation Equity Act for the 21st Century) as a successor project 
ISTEA was formulated in 1997s. Compared with ISTEA, TEA-21s project scale and economic investment have a significant growth which can provide a strong guarantee for the development of ITS technology. In order to improve the safety and efficiency of the nations road transportation system, Federal and state departments of transportation (DOTs) cooperated with vehicle manufacturers to propose the Vehicle Infrastructure Integration (VII)[3] based on the evaluation of the technical, economical, and political feasibility of deploying a communications system. The VII provides a communication link between vehicles on the road (via On-Board Equipment, OBE), and also between vehicles and the roadside infrastructure (via Roadside Equipment, RSE). The VIIs key technology is emphasized on short range communication (DSRC) which is allocated by FCC, and spans over $75 \mathrm{MHz}$ of spectrum in the 5.9 $\mathrm{GHz}$ band in US. VII connects vehicles and infrastructure and creates an enabling communication infrastructure. Implementation of ITS in the United States varies immensely by state and region, thus tend ing to be sporadic, isolated, and, unlike Japans Smartway, not connected into a nationally integrated intelligent transportation system.But there are two base premises that all new vehicles would be equipped with DSRC at $5.9 \mathrm{GHz}$ and GPS and a nationwide roadway-based communications network should be created. Fig.3 shows the Architecture of VII

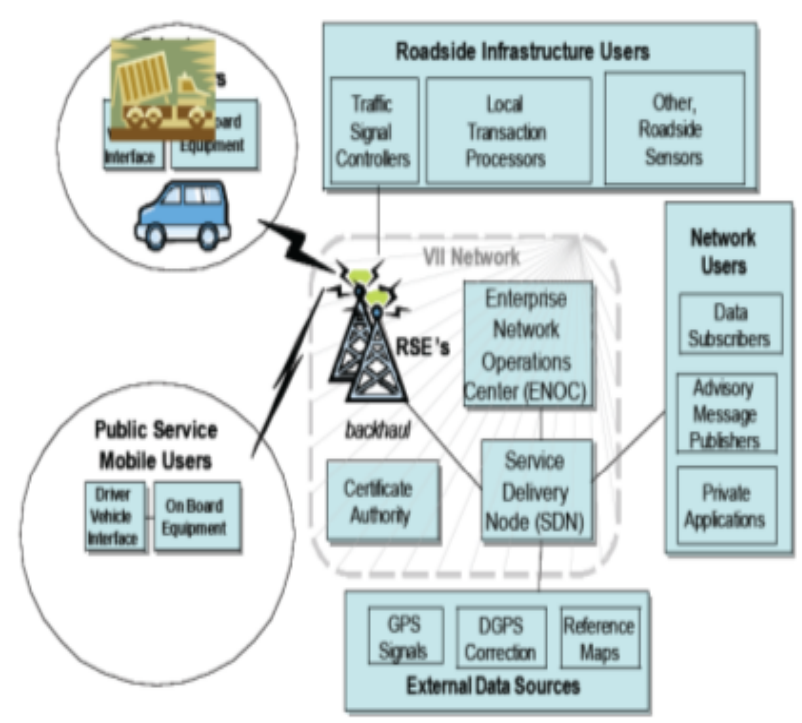

Fig. 3. Vehicle Infrastructure Integration Architecture

\subsection{Singapore}

Singapore is one of the world leaders in development of Intelligent Transportation Systems based on its:

(1) Use of probes vehicles to collect real-time traffic information,

(2) Use of electronic road pricing (that is, congestion charging) and

(3) Nationwide deployment of adaptive computerized traffic signals.

Singapores Land Transport Authority (LTA)[4] is the organization responsible for all modes of transportation in the country and oversees implementation of Intelligent Transportation Systems in Sin-

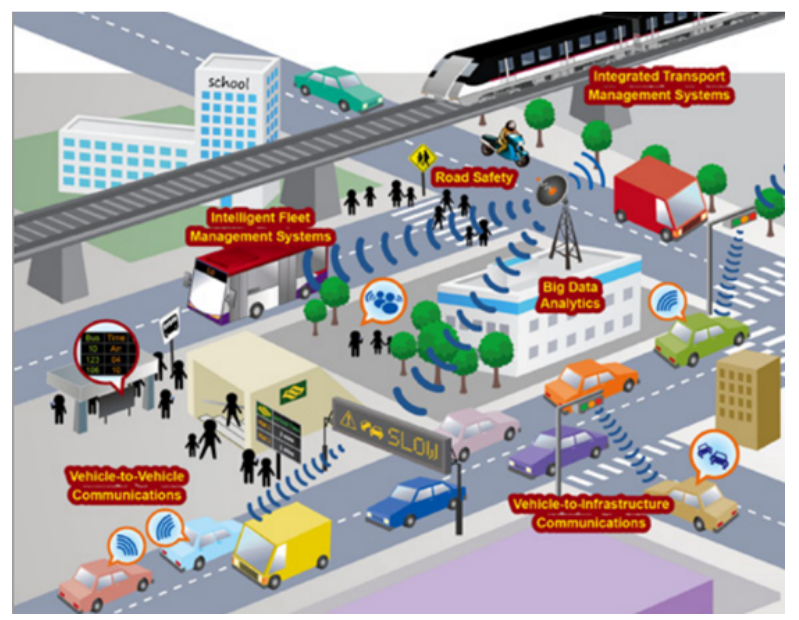

Fig. 4. Singapore ITS implementation plan

gapore. The country's ITS Master Plan aims to create an optimized and efficient land transport network leveraging ITS to enhance commuters travelling experience. Singapore collects realtime traffic information through a fleet of 5,000 taxis running on the roads which act as vehicle probes, sending their speed and location information back to Singapore's Traffic Operations Management department, enabling it to generate an accurate picture of traffic flow,patterns and congestion on Singapores roadways from this critical mass of probe data. Singapore has a commercial agreement with the taxi operators. Singapore has implemented a fully automated electronic road pricing (ERP) system that basically uses DSRC with an On-Board equipment installed in each car that accepts a prepaid stored-value smart card called as the Cashcard. Singapore uses a very intelligent and novel method to automatically deduce the amount of using a particular road from the Cashcard when the vehicle passes through an ERP gantry. Singapore's ERP scheme actually uses traffic speeds as a proxy for congestion. Rates are raised or lowered to achieve traffic optimization along a speedflow curve, 45 to $65 \mathrm{kmph}$ for expressways and 20 to $30 \mathrm{kmph}$ for arterial roads. In nutshell, the system uses market signals to manage supply and demand on Singapores roadways.

\section{KEY UNDERLYING TECHNOLOGIES}

\subsection{GPS Reciever Module}

GPS receiver receives the signal sent by the GPS[5] satellites. In our system GPS receiver module is used to get the position and speed of the bus. The receivers cannot transmit and while receiving the satellite signals the GPS receivers get the signals passively. It requires a clear perspective of the sky and hence would be less efficacious indoors. So they are generally placed outside the bus such that it gets a clear view. The time reference required for GPS is administrated by an atomic clock. These are placed on the satellites and are very accurate. The data send by each satellite includes its current position and time. To transmit the signals from different satellites at the same time, the operations of the GPS satellites are synchronized. Some satellites are near and others far. Hence the signals reach at different instants at the receiver. The signals travel at the speed of light. 


\subsection{GSM/GPRS Modem}

GSM/GPRS RS232 Modem from rhydoLABZ is built with SIMCOM Make SIM900 Quad-band GSM/GPRS engine. It works on frequencies $850 \mathrm{MHz}, 900 \mathrm{MHz}, 1800 \mathrm{MHz}$ and $1900 \mathrm{MHz}$. This module is used for the communication of different modules of the architecture. Basically it is the medium for the flow of information from one module to another. AT commands are used to send commands for communication purposes . AT stands for attention. It is used to send SMS between the different sections. SMS can also be read using AT commands. It also can perform many other functions. The required data for each section is transmitted from one section to another through this modem. It is suitable for SMS as well as DATA transfer application in M2M inter- face. The modem requires mainly three wires, Tx (transmit), Rx (receive), and GND (ground).

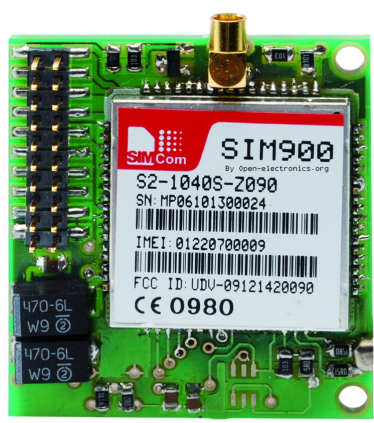

Fig. 5. GSM SIM900 Module

\subsection{Dedicated-Short Range Communications(DSRC)}

Dedicated-Short Range Communications (DSRC) is one- way or two-way wireless communication channel that operates in the 5.8 or $5.9 \mathrm{GHz}$ wireless spectrum and are specifically designed for automotive uses. DSRC is capable for two-way wireless communications between the vehicle and roadside equipments. It plays a vital role in many intelligent transportation systems including vehicle-to-infrastructure integration, vehicle-to-vehicle communication, adaptive traffic signal timing, electronic toll collection, congestion charging, electronic road pricing, information provision, etc. DSRC is a subset of radio frequency identification (RFID) technology . The technology for ITS applications works on the $5.9 \mathrm{GHz}$ band in United States and the $5.8 \mathrm{GHz}$ band in Japan and Europe. Nowadays, DSRC systems in Europe, Japan and the United States are generally not compatible. In 2004, the U.S. Federal Communications Commission (FCC), prescribed a common standard for the DSRC band both to promote interoperability and to discourage the limitation of competition through proprietary technologies .

\subsection{Wireless networks}

Wireless networks allow rapid communication between vehicles and the roadside which is similar to technology commonly used for wireless Internet access, however this technology is limited to a range of only a few hundred meters. Nevertheless, this range can be extended to pass information onto the next vehicle or node by each successive vehicle or roadside node. WiBro is increasingly in usage in South Korea, based on WiMAX technology, as the wireless

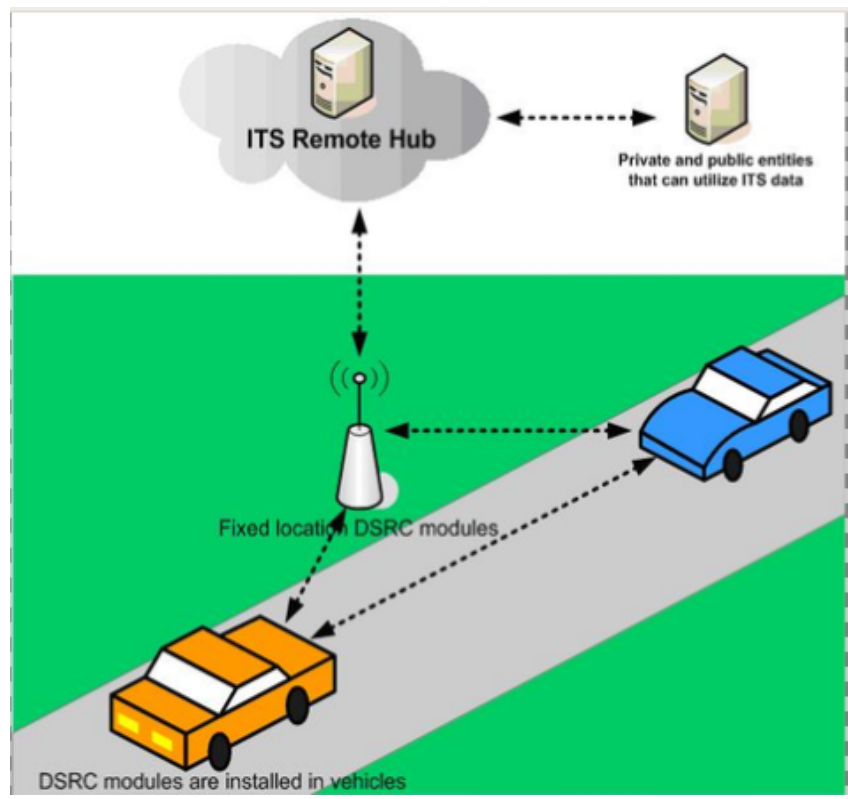

Fig. 6. Basic Working of DSRC

communications in frastructure to transmit traffic and public transit information throughout its transportation network.

\subsection{Roadside Camera Recognition}

Camera or tagging-based schemes can be used for zone-based or area-based congestion charging systems (as in London), as well as for charging on specific routes. High speed cameras are placed on specific location on roadways mostly where drivers enter and exit congestion zones in such systems. The cameras utilize the technology called Automatic License Plate Recognition (ALPR) to identify vehicle license plates which is based on Optical Character Recognition (OCR)[6] technology. The back-office servers process the data, perform analysis and post charges to drivers for their use of roadways within the congestion zone. Moreover, it is able to pass the information digitally in a convenient manner[7]

\subsection{Probe Vehicles or Devices}

The so-called probe vehicles (often taxis or government owned vehicles equipped with DSRC or other wireless technology) are being deployed by several countries. These probes are basically transmission units which collect extensive information about the speed and location of vehicles and transmit it to a central traffic operations management center, where this data is received, aggregated, processed to generate an area-wide picture of traffic flow and to evaluate congested locations. Extensive research is also being carried out to use mobile phones that drivers often carry on a regular basis as a useful mechanism to generate real-time traffic information, using the GPS-derived location of the phone as it moves along with the vehicle. For example, in Beijing, more than 10,000 taxis and commercial vehicles have been equipped with GPS chips that send travel speed information to a satellite, which then sends the information down to the Beijing Transportation Information Center and then the data is transformed into average travel speeds on every road in the city [7]. 


\section{PROPOSED ARCHITECTURE}

The proposed system consists of a OnBoard Unit, a GPRS/GSM based transmiter module, a receiver and display module for public which provides information about the arrival and departure time of the buses and a client side module. This section proposes a system which could track the current position of buses and the dynamic arrival and departure time and inform the passengers via display boards at the terminus or through an App installed in a smart phone or through SMS for normal phones. The transmitter module is fitted with the bus and the receiver module at the bus terminus. Many transmitter modules can communicate with a single receiver module at the bus terminus. The latitude and the longitude coordinates of the bus are transmitted to the bus terminus via GSM.

\subsection{Bus Module}

Bus module is introduced inside every bus and consists of a GSM/GPRS modem, digital speedometer, SD card, RTC, GPS and LED matrix display all of which is interfaced to the microcontroller (Arduino Mega). Through GPS the current location and speed, and from RTC, time and date is obtained. This information is send to the server using GSM/GPRS modem. The speed information is used to check whether the vehicle exceeds the threshold speed. In case of unavailability of GPS, a digital speedometer is used to calculate the speed. If the threshold speed is exceeded, the speed, date and time are stored in SD card for future reference. Also an indication of over speed is displayed on LED matrix for the notification of the passengers . The next stop indicator helps the passengers to understand the route and the current position of the bus. The bus stops are predefined in the microcontroller EPROM. When the bus arrives at the bus stop, the next approaching bus stop name is displayed on the LED matrix as well announced using a speaker in native languages. This will help the passenger if he is doubtful about the exact location of his destination.
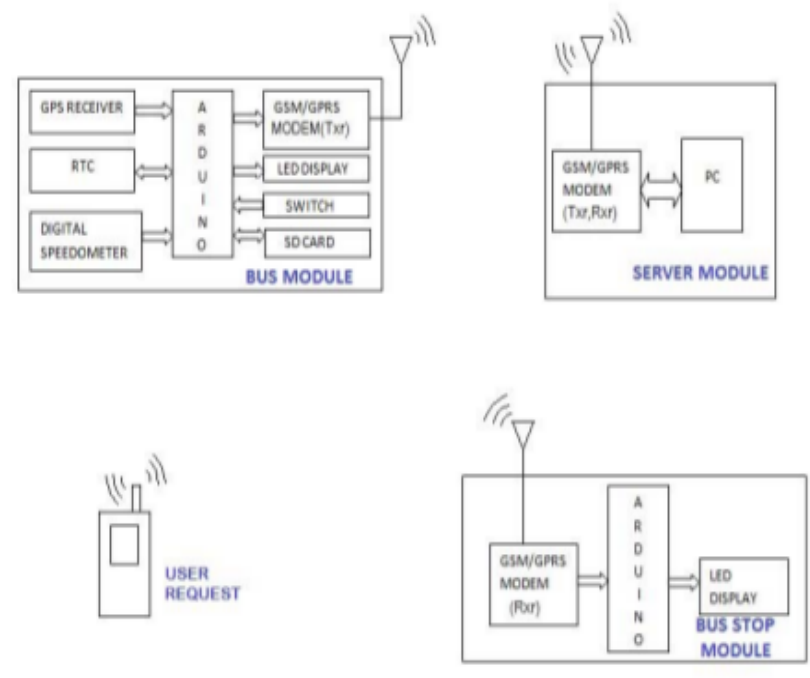

Fig. 7. Block diagram representation of the proposed architecture

\subsection{Base Station/Server Module}

The server module sits at the centre of this architecture. It serves as the back-end tool. The server contains all the information about the routes of all the buses as well as the intermediate bus stops on the route. The server also processes request from a client regarding the estimated arrival time of a bus at a particular bus stop.The server maintains a database of information pertaining to the buses, routes and stops in the form of tables on a DBMS like MYSQL. The server database can be organized in many ways, to reduce memory requirement, improve access speed, or reduce the number of queries. To improve the query response mechanism and to reduce the query reply time, the tables related to buses are partitioned into static and dynamic ones. The Bus table stores all the static data like bus stops on a route, while the bus position and log tables store dynamic data. The relation and dependencies between the unique bus id, bus type and route number is stored in the Bus table. The position updates from the bus are stored in the Bus Position column and the Bus Position Log tables. The direction field helps us in finding the direction in which the bus is headed (Terminus A to Terminus $\mathrm{B}$, or reverse). The direction is calculated in the vehicle unit by comparing the order of the bus-stops appearance on the route. The average speed is the weighted average of the current speed and the previous average speed.

\subsection{Bus Terminus Controller Environment (BTCE)}

This consists of a GSM module which receives the co-ordinates of the position of the bus and passes it to the Arduino board. The microcontroller of the Arduino processes this data and calculates the exact position of the bus travelling towards the terminus and the time it will take to reach the terminus. These timings are stored in the server and available for access by the passengers via their smart phone app through internet as well as through SMS. At the same time these timings are also displayed on the display board for the comfort of the passengers who are yet waiting for the bus to arrive. If a passenger can know the timings of the arrival of buses, his or her anxiety is greatly reduced and can relax. The commuters at home can check the live updates of the position and time of arrival of a particular bus at the terminus via either smart-phone app or SMS . Apart from the arrival time, the BTCE would be able to identify the delay time too using a simple algorithm and update the users regarding the same.

\subsection{Participatory Sensing}

Also known as Moble Crowd Sensing(MCS) is a very interesting and innovative idea of making the Public Transport System efficient using the power and cooperation of the general public. It uses the power of crowd in the bus and on the bus stops for gathering required information about the buses. It is an alternative to the traditional GPS-based bus tracking system. In cases where GPS data is not available this concept can prove to very handy and effective. As it is not using GPS ,the system is very cost effective. In this system no particular hardware is required. Every thing is done using passengers phone. Though sensors can be used to provide real-time traffic data but nothing can surpass the power of people travelling in the bus to transmit this information. The commuters have a better idea regarding unexpected delays, traffic jams, capacity of bus which can prove to be very useful to other passengers also. For delivering the service information to passengers QR codes can be used. QR codes are easy to create and use. They can be placed on the bus stops. The passengers then can scan the QR code(with their smart phones) and get the required information about the buses,routes,etc. The main challenge in this system is motivating the passengers for their involvement. But once it is done then in a very low cost, an effective bus transport management system can be built. 


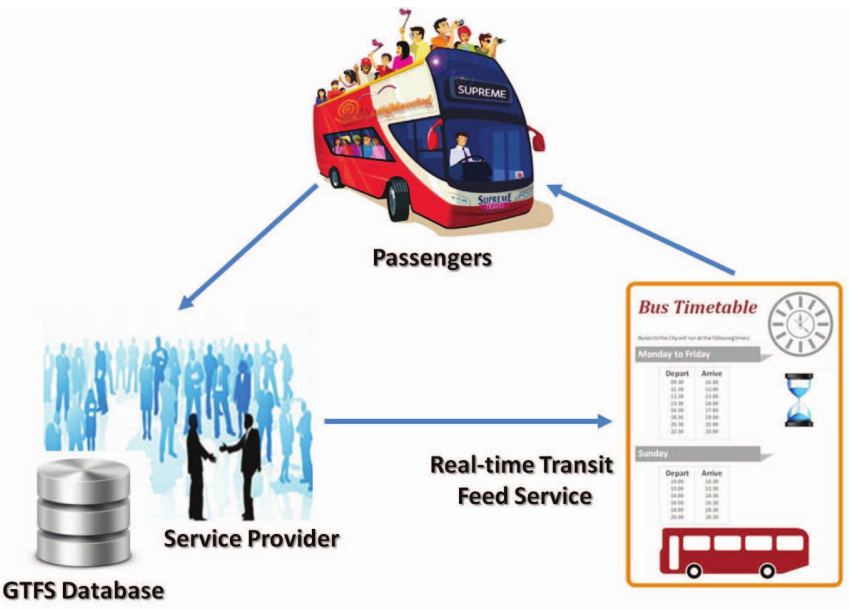

Fig. 8. Participatory sensing Architecture

\section{CONCLUSION}

Traffic management when done effectively, can improve the state of the country drastically. It not only will cause improvement in the environmental conditions like decrease in pollution ,less congestion ,etc. but also contribute a big deal in the economic growth of the country. We regard the traffic management as not only the problem of an individual country, but as a global issue which needs to be tackled in a tactful manner . The paper has proposed an Intelligent Public transport Management System that tracks the current location of all the buses and estimates their arrival time at different bus stops in their respective routes. Estimates are updated every time the bus sends an update. It distributes this information to passengers using display terminals at bus stops, web based GUI, SMS and smartphone apps. This system serves the needs of passengers, vehicle drivers and administrators of the transport system in a very useful manner .

\section{FUTURE WORK}

The proposed system is useful for a wide range of population, as it serves the section of society who possess use buses on a regular basis, by providing details on smartphones, as well to those who do not own a smart phone, by displaying information on the display panels erected on the bus stops and SMS service. This system is hardware based which has a few limitations like hardware failure, malfunctioning etc. To overcome these problems, a more robust solution using a combination of better and faster components can be implemented. There is also scope for implementing context aware driver behavior system in buses to avoid accidents and reduce deaths caused to rash and irrational driver behavior.

\section{REFERENCES}

[1] Japan highway industry development organization. ITS Handbook Japan, 2007-2008.

[2] Pham Hoang Oat Micheal Drieberg and Nguyen Chi Cuong. Development of vehicle tracking system using gps and gsm modem. In IEEE Conference on Open Systems (ICOS), pages 89-94. IEEE Conference Publications, 2013.

[3] A. EI-Rabbany. Introduction to GPS: The Global Positioning System, chapter GPS for Vehicle Navigation, pages 8399. Norwood, MA: Artech House, 2006.
[4] Setphen Ezell. Intelligent Transportation Systems, pages 140. January 2010.

[5] Sheng hai An Byung-Hyug Lee and Dong-Ryeol Shin. A survey of intelligent transportation systems. In CICSYN '11 Proceedings of the 2011 Third International Conference on Computational Intelligence, Communication Systems and Networks, pages 332 - 337. Conference Publishing Services, 2011.

[6] How VICS Works Available online at :. http://www.vics.or.jp/en/vics/.

[7] Intelligent Transport Systems Available online at :

[8] Intelligent Transportation Systems Joint Program Office Available online at :. http://www.its.dot.gov/vii/.

[9] Alwyn Antony Ancy Devassy Jinesh K J Rose Mary John Finky Francis Joe Neelankavil. Smart public transport system. In 2014 International Conference on Embedded Systems ICES 2014, pages 167-170, 2014.

[10] What is OCR and OCR Technology Available online at : . http://www.abbyy.com/finereader/aboutocr/what-is-ocr/. 